\title{
A Way to Estimate the Discharge of the Melt Jet Flowing out of a Melting Furnace
}

\author{
V. N. Kruglov and A. V. Kruglov \\ Ural Federal University named after the first president of Russia B.N. Yeltsin, ul. Mira 19, Yekaterinburg, 620002 Russia \\ e-mail:v.krouglov@mail.ru
}

\begin{abstract}
A way to estimate the discharge of melt jet flowing out of a melting furnace is examined. We analyze the efficiency of algorithms for image matching in order to estimate the jet velocity. To decrease the time needed for estimating the 2D motion of melt flow, it is suggested to calculate the shift of $2 \mathrm{D}$ images according to two independent $1 \mathrm{D}$ projections. This approach makes it possible to estimate on-line the flow rate and to do it in television standard.
\end{abstract}

Keywords: a system of technical vision, melting furnace, melt jet discharge, image matching, image digital processing, cyclic invariants.

DOI: $10.1134 / \mathrm{S} 1054661813040081$

\section{INTRODUCTION}

The modern industry of mineral wool production is characterized by high automation. All processes of raw material and fuel feeding including components completing, weighing, transportation, and feeding into a furnace are automated and performed according to a program. Special sensors determine and control the centrifuge's rotary speed, blowing temperature and humidity, and gas output. But several problems have not yet been solved, for example, the problem of how to consider the melt flow rate. Ordinary procedures for measuring the liquid flow rate are not suitable, since the medium is corrosive. The existing procedures for determining the melt flow rate are not efficient enough and do not meet the requirements on planning production cost. However, continuous checking on the melt flow rate is one of the most important problems for mineral wool production, since if the flow rate is overvalued, the raw materials and fuel are excessive, and if the flow rate is undervalued, the production quality drops. In any case, a sharp variation in melt flow rate is a symptom of any failure dealing with furnace operation. The melt flow rate should be monitored continuously making it possible to increase the production quality.

\section{THE MAIN PART}

To calculate the volume of material flowing out of the melting furnace for a certain period of time (melt discharge), it is necessary to know the material rate of motion within this period of time and the flow cross section. The problem on how to estimate the material rate of motion can be solved by a procedure for image recording. Under image recording we mean the process for determining the fragment position of one image in another one.

Let $f_{M}^{e}(x, y)$ be the image of material flow at time moment $t_{0}$. Let one of its $N \times N$ fragments, in particular, $f_{N}^{e}\left(x_{e}, y_{e}\right)=W_{N}\left(x-x_{e}, y-y_{e}\right) f_{M}^{e}(x, y)$ contain an image of the greatest relief segment of material flow, the coordinates of which coincide with the fragment center $\left(x_{e}, y_{e}\right)$; we call it a reference fragment (Fig. 1).

In the next frame, we call it the current one $f_{M}^{c}(x, y)$; at the time moment $t_{1}$ the center of reference fragment

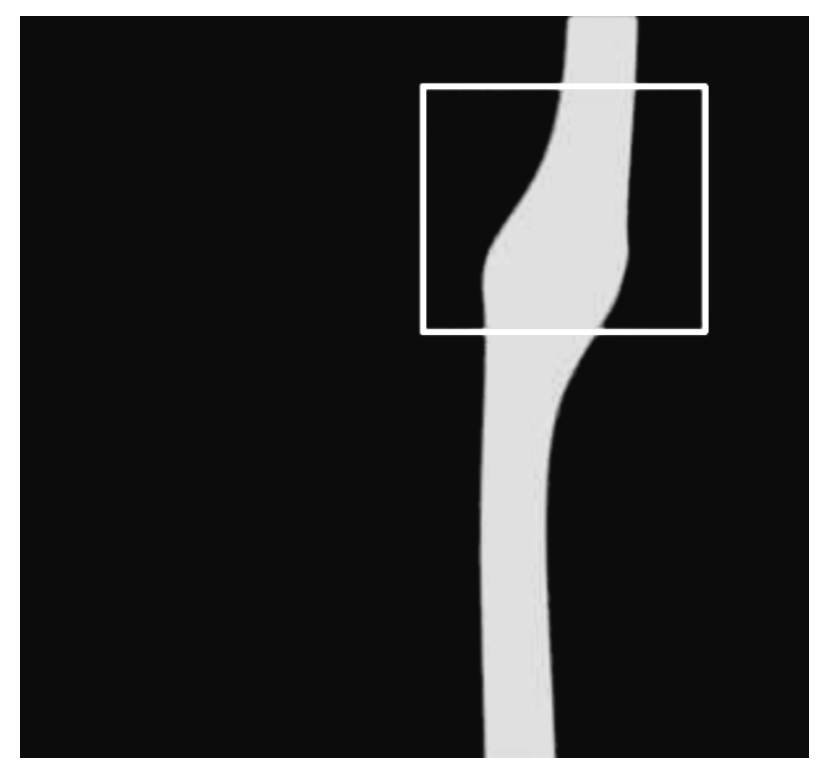

Fig. 1. Reference fragment of material flow. 


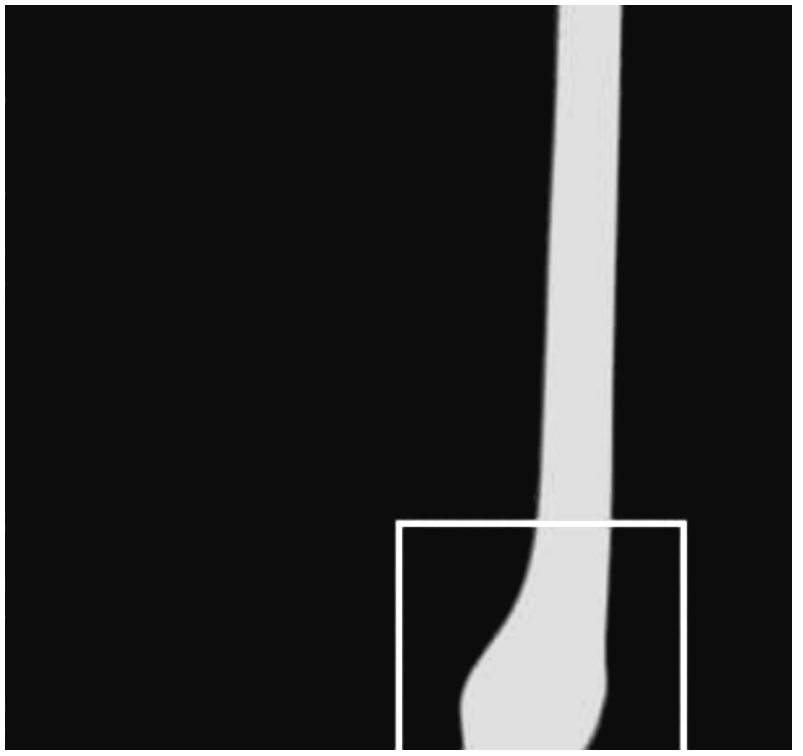

Fig. 2. Current fragment of material flow.

$f_{N}^{e}\left(x_{e}, y_{e}\right)$ travels from point $\left(x_{e}, y_{e}\right)$ into another one, for example, into point $\left(u^{*}, v^{*}\right)$.

The procedure for image recording is as follows. The search for the $N \times N$ window $W_{N}$ is scanned according to $f_{M}^{c}(x, y)$. In each position $W_{N}$ separates fragment $f_{N}^{c}(u, v)=W_{N}(x-u, y-v) f_{M}^{c}(x, y)$ from the current image, for which the similarity with reference fragment $f_{N}^{e}\left(x_{e}, y_{e}\right)$ is determined. The position $\left(u^{*}, v^{*}\right)$ of the search window $W_{N}\left(x-u^{*}, y-v^{*}\right)$ under which there is the greatest similarity between $f_{N}^{c}\left(u^{*}, V^{*}\right)$ and $f_{N}^{e}\left(x_{e}, y_{e}\right)$ makes it possible to determine the shift value of the reference fragment and the rate of motion for the material flow.

We reviewed algorithms for images matching with respect to the examined scientific field, in particular, for estimating the jet motion between two neighboring video frames, and we revealed that the most promising in terms of matching accuracy and computation complexity was the set of algorithms based on the measure of similarity $\mu$ between the reference $f_{N}^{e}(x, y)$ and current $f_{N}^{c}(u, v)$ signals. It was calculated by using the absolute difference method (MAD) [1]:

$$
\mu\left[f_{e}, f_{c}, u, v\right]=\sum_{x=0}^{N-1} \sum_{y=0}^{N-1}\left|f_{N}^{e}(x, y)-f_{N}^{c}(u, v)\right| .
$$

The modifications of this algorithm are as follows: the optimized strategies for searching for a global extremum for the measure of similarity are different [1-3]. Such strategies are three-step search, hexagonal search, and cross-hexagonal search.

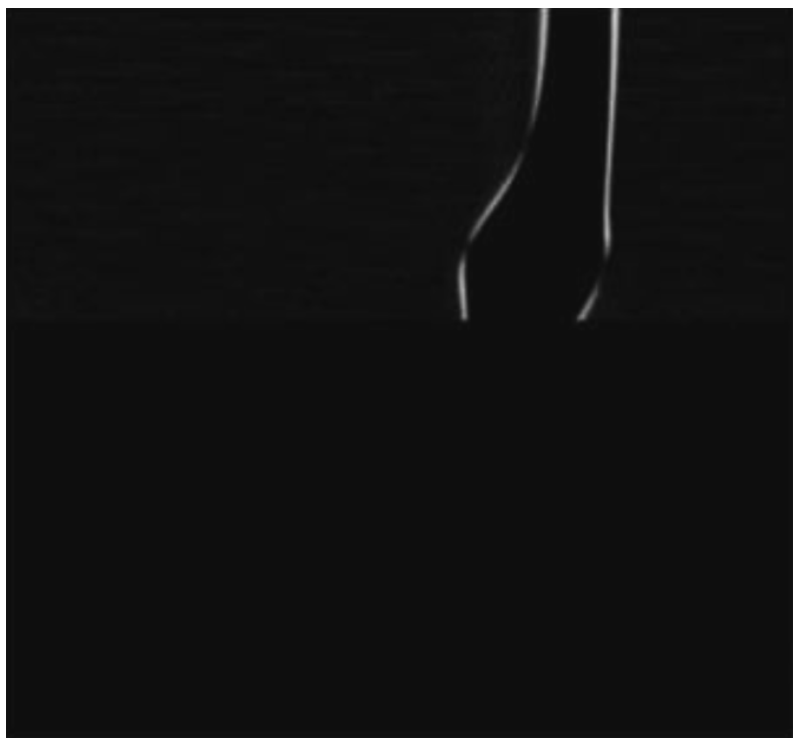

Fig. 3. Visualization of test image matching performed by hand.

To determine the accuracy of shift estimation, we programmed and simulated these algorithms mathematically. As test images we choose three typical subjects that present images of two sequential video frames (previous and current) of melt jet running out of the melting furnace. One such subject is presented in Figs. 1 and 2.

To verify the algorithms, we develop additional software that makes it possible to estimate by hand the shift of the jet image between two neighbor frames.

Figure 3 depicts the results of video frame matching performed by hand.

The quantitative characteristics obtained according to the experimental results are presented in the table. The size of the processed video frames is $768 \times$ 576 pixels, and the size of the reference image is $250 \times$ 250 pixels. The time of algorithm operation is averaged, since the OS WINDOWS is not an on-line operating system and it is impossible to measure exactly the time for executing one algorithm or another.

The examined procedures for estimating the shift of image $f_{N}^{c}(u, v)$ with respect to image $f_{N}^{e}(x, y)$ are $2 \mathrm{D}$ procedures. That is why their computation complexity is significantly higher than the computation complexity intrinsic to their $1 \mathrm{D}$ analog. Due to this fact, it is of interest to develop quick algorithms for estimating the shift of $2 \mathrm{D}$ images, the computation burden of which is close to the computational burden intrinsic to the $1 \mathrm{D}$ algorithm.

To solve this problem, it is suggested to use a method for separating the 2D shift onto two independent $1 \mathrm{D}$ ones that makes it possible to process $1 \mathrm{D}$ arrays instead of $2 \mathrm{D}$ ones.

The separation is performed by calculating the cyclic invariants for each line and each column (a kind 
The results of algorithm simulation for images matching according to the measure of similarity MAD with different strategies for searching for a global extremum

\begin{tabular}{|c|c|c|c|c|c|c|}
\hline \multirow{2}{*}{$\begin{array}{c}\text { Strategy } \\
\text { for searching for } \\
\text { a global extremum }\end{array}$} & \multirow{2}{*}{ Tested flow } & \multicolumn{2}{|c|}{$\begin{array}{l}\text { The way to estimate the video } \\
\text { frame shift for a jet flow }\end{array}$} & \multicolumn{2}{|c|}{ The error of algorithm operation } & \multirow{2}{*}{$\begin{array}{c}\text { The time } \\
\text { of algorithm } \\
\text { operation }(\mathrm{ms})\end{array}$} \\
\hline & & by hand (pixels) & algorithmic (pixels) & absolute (pixels) & relative $\%$ & \\
\hline \multirow[t]{3}{*}{ Total search } & 1 & 333 & 334 & 1 & 0.3 & 4073 \\
\hline & 2 & 328 & 328 & 0 & 0 & \\
\hline & 3 & 340 & 342 & 2 & 0.58 & \\
\hline \multirow[t]{3}{*}{ Three-step search } & 1 & 333 & 318 & 15 & 4.5 & 79 \\
\hline & 2 & 328 & 340 & 12 & 3.66 & \\
\hline & 3 & 340 & 352 & 12 & 3.53 & \\
\hline \multirow[t]{3}{*}{ Hexagonal search } & 1 & 333 & 326 & 7 & 2.1 & 53 \\
\hline & 2 & 328 & 320 & 8 & 2.44 & \\
\hline & 3 & 340 & 335 & 5 & 1.47 & \\
\hline \multirow{3}{*}{$\begin{array}{l}\text { Cross-hexagonal } \\
\text { search }\end{array}$} & 1 & 333 & 336 & 3 & 0.9 & 28 \\
\hline & 2 & 328 & 326 & 2 & 0.61 & \\
\hline & 3 & 340 & 344 & 4 & 1.18 & \\
\hline
\end{tabular}

of generalized projection (shadows) of the 2D image onto two mutually normal directions). A particular case of this method is the procedure for generating an ordinary "shadow," to summarize all elements for lines and columns.

Let $f(x, y)$ be the brightness function of a certain image, $x, y=\overline{0, N-1}, S(\cdot, \cdot, \ldots, \cdot)$ be a symmetric function with respect to $N$ variables (for example, $N-1$

$\left.\sum_{x=0} \bullet\right)$, and $\left\{U_{\omega}(\bullet, \bullet, \ldots, \bullet) \mid \omega=\overline{1, K}\right\}$ be an arbitrary set of $K$ functions with respect to $l$ variables. Let us take $l$ arbitrary horizontal countings $f\left(x_{1}, y\right), f\left(x_{2}, y\right), \ldots$, $f\left(x_{l}, y\right)$, and let us calculate as follows:

$$
\begin{gathered}
I_{\omega}^{y}(f(0, y), f(1, y), \ldots, f(N-1, y)) \underline{\underline{\Delta}} S \\
\times U_{\omega}\left[f\left(x_{1}, y\right), f\left(x_{2}, y\right), \ldots, f\left(x_{l}, y\right)\right], \\
U_{\omega}\left[f\left(x_{1} \oplus 1, y\right), f\left(x_{2} \oplus 1, y\right), \ldots, f\left(x_{l} \oplus 1, y\right)\right], \ldots, \\
U_{\omega}\left[f\left(x_{1} \oplus(N-1), y\right), f\left(x_{2} \oplus(N-1), y\right), \ldots,\right. \\
\left.f\left(x_{l} \oplus(N-1), y\right)\right],
\end{gathered}
$$

where $\oplus$ means summarizing over $\bmod N$. If the whole array $\{f(0, y), f(1, y), \ldots, f(N-1, y)\}$ is shifted cyclically, there is change of variables $U_{\omega}[\ldots]$ inside symmetrical function $S$, which does not change its value. The value

$$
I_{\omega}^{y} \triangleq I_{\omega}(f(x, y))=I_{\omega}(f(0, y), f(1, y), \ldots, f(N-1, y))
$$

we call an invariant of $y$-th column for image $f(x, y)$. A set of invariants $I_{\omega}^{y}$ under $\omega=$ const, $y=\overline{O, N-1}$ forms a $1 \mathrm{D}$ array, a line, and we call it an invariant pro- jection for columns with number $\omega$. For a similar invariant line projection, we use the designation $I_{\omega}^{x}$.

\section{CONCLUSIONS}

If we separate the motion of the $2 \mathrm{D}$ object into mutually orthogonal directions, it becomes possible to estimate the shift by means of the 1D method, for example, MAD, independently of each other. If we use this approach for estimating the rate of motion for the jet flowing out of the melting furnace, it makes possible to reduce the time of algorithm operation down to $8 \mathrm{~ms}$, to estimate on-line the melt discharge, and to do it in television standard.

\section{REFERENCES}

1. Ishfaq Ahmad, Weiguo Zheng, Jiancong Luo, and Ming Liou, "A fast adaptive motion estimation algorithm," IEEE Trans. Circuits Syst. Video Techn. 16 (3), 420-438 (2006).

2. Yair Moshe and Hagit Hel-Or, "Video block motion estimation based on gray-code kernels," IEEE Trans. Image Processing 18 (10), 2243-2254 (2009).

3. Yih-Chuan Lin and Shen-Chuan Tai, "Fast full-search block-matching algorithm for motion-compensated video compression," IEEE Trans. Commun. 45 (5), 527-531 (1997).

4. B. Sirok and B. Blagojevich, Mineral Wool (Woodhead Publ., Cambridge, 2008), p. 184.

Translated by Yu. Zikeeva 


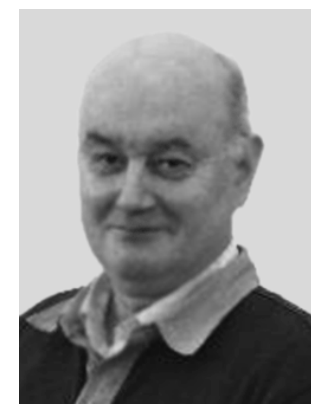

Vasilii N. Kruglov. Graduated from the Kirov Ural Polytechnic Institute in 1977 (radio engineering department, specialty Automation for control systems). Candidate of technical science since 1989. Fields of interest: systems of technical vision: development, manufacturing and introduction in to mining and metallurgy enterprisers. In 2005 Kruglov developed the television computer system "Size Indicator" for checking the granulometric composition of milled ore and introduced it at the Mikhailovsk ore-dressing and processing enterprise. In 2006 he developed (jointly with NPVP TOREKS Company) a system for controlling the cup-type pelletizer for producing raw rolls and introduced it at the Lebedinsk ore-dressing and processing enterprise (Staryi Oskol). In 2007 he developed the television computer system "Indicator for Jet Discharge" for estimating the melt flow rate of a small melting furnace for mineral wool production and introduced it at the AKSI enterpriser (Chelyabinsk).

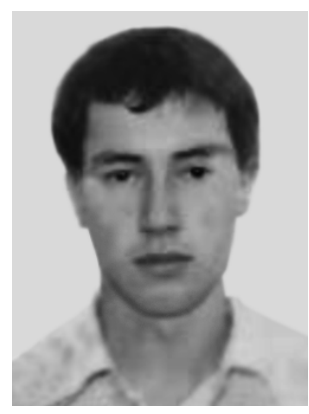

Artem V. Kruglov. Received bachelor of engineering and technology (specialty information and computer sciences (Ural Federal University). Presently studying for master's degree (specialty Information and Control Systems). Fields of interest: computer vision, image recognition and reconstruction, automated control systems. At the present time he is a director of a company designing control systems based on technical vision. 